\title{
Nanostructured Silicon Photo-Cathodes for X-Ray Generation
}

\author{
M. E. Swanwick ${ }^{1 *}$, P. D. Keathley ${ }^{2}$, F. X. Kärtner ${ }^{2,3}$, and L. F. Velásquez-García ${ }^{1}$ \\ ${ }^{1}$ Microsystems Technology Laboratories, Massachusetts Institute of Technology (MIT), Cambridge, MA, USA \\ ${ }^{2}$ Dept. of Electrical Engineering and Computer Science and Research Laboratory of Electronics, MIT \\ ${ }^{3}$ Center for Free-Electron Laser Science, DESY and Dept. of Physics, University of Hamburg, Hamburg, Germany \\ *swanwick@mit.edu
}

\begin{abstract}
We report the fabrication and characterization of ultrafast laser triggered nanostructured silicon photo-cathodes for $x$-ray generation via inverse Compton scattering. A highly uniform array of $\sim 2200$ silicon pillars with $5 \mu \mathrm{m}$ array pitch, where each pillar is capped by a nanosharp tip, shows stable current emission using $35 \mathrm{fs}, 800 \mathrm{~nm}$ laser pulses. The cathodes can emit at 3.6 $\mathrm{nA}$ average current over 8-million $1.2 \mathrm{pC}$ electron bunches when excited with $9.5 \mu \mathrm{J}$ laser pulses with no degradation of the emission characteristic of the cathode, showing that silicon-based photon-triggered cathodes processed with standard CMOS processes and operated at high vacuum can function for extended periods without performance degradation.
\end{abstract}

Keywords- coherent x-ray; field emission; MEMS; multiplexed; photocathode; ultrafast optics

\section{INTRODUCTION}

Nanostructured cathodes that can be switched at an ultrafast time scale $(<1 \mathrm{~ns})$ have applications in free electron lasers and coherent x-ray sources [1]. State-of-the-art ultrafast cathodes are flat surfaces that emit electrons using ultraviolet (UV) pulses, harnessing the photoelectric effect; these devices need ultra-high vacuum to operate and have short lifetime. Photontriggered field emission cathodes are an attractive alternative to circumvent the shortcomings of UV photocathodes, while providing a direct means of structuring the emitted electron beam. Narrow tip radii distributions are required to achieve high utilization of the field emitter array given the exponential dependence of the field-emitted current on the emitter field factor. For coherent x-ray generation to be possible, the photocathode needs to produce a uniform series of nanostructured charge sheets with sufficient charge over an extended period of time.

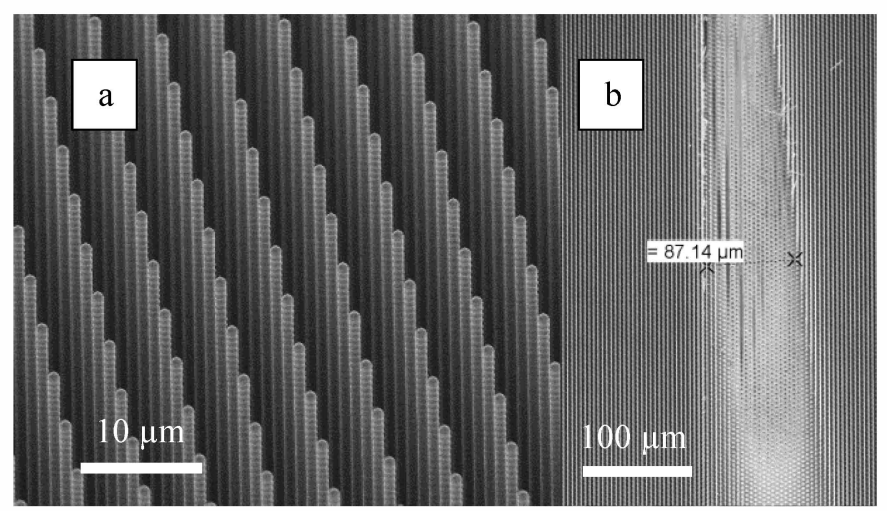

Fig. 1 - (a) SEM of high aspect-ratio single-crystal Si pillars with uniform nanosharp tips. (b) Damage to Si tips after exposed to $20.5 \mu \mathrm{J}$ laser pulses.

\section{FABRICATION}

The cathodes are fabricated from single-crystal $<100>\mathrm{n}-\mathrm{Si}$ 1-10 $\Omega$-cm wafers. The process flow includes the fabrication of arrays of hard mask features patterned by projection optical lithography and reactive ion etching; each feature of the array is a silicon dioxide disk on top of a concentric silicon nitride disk. The substrate is then deep reactive-ion etched to form arrays of high-aspect-ratio silicon pillars. Next, the substrate is oxidized to further thin the pillars and to create a nanosharp tip on top of each pillar; finally, the oxide and nitride are striped by selective wet processing. The result are massive arrays of pillars (over half a million elements with $5 \mu \mathrm{m}$ hexagonal packing) capped by tips with $5 \mathrm{~nm}$ average tip radius and less than $1 \mathrm{~nm}$ standard deviation [2] (Fig. 1a).

\section{APPARATUS AND MEASUREMENT PROCEDURE}

The cathodes are photo excited using $35 \mathrm{fs} 800 \mathrm{~nm}$ pulses at a $3 \mathrm{kHz}$ repetition rate from a titanium sapphire laser at $84^{\circ}$ glancing angle, inside a vacuum chamber at $\sim 10^{-8}$ torr. The beam spot floods $\sim 2220$ tips. The cathode chip is connected to ground through a pico-ammeter while the anode, i.e., a 0.25 " plate $4 \mathrm{~mm}$ above the cathode, is connected to a voltage supply (Fig. 2).

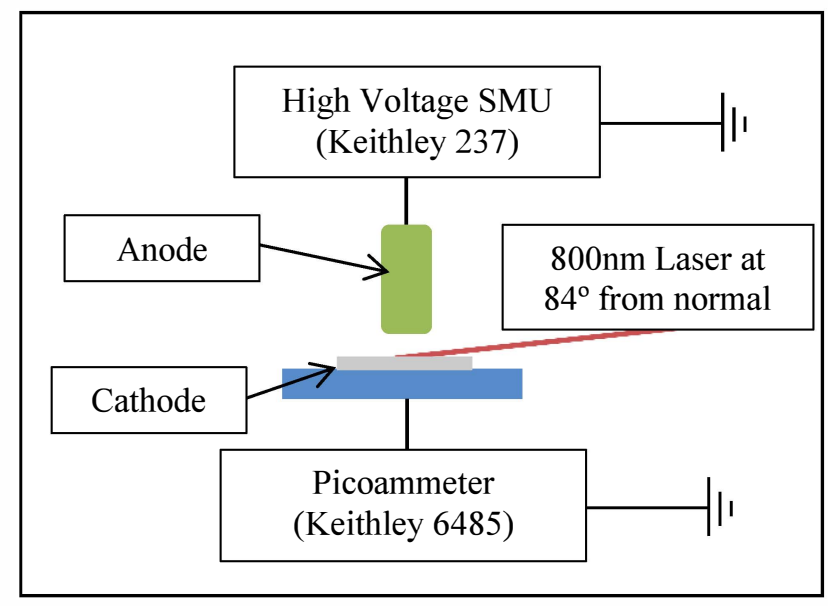

Fig. 2 - Schematic of testing chamber with the cathode connected to ground through a picoammeter (Keithley 6485 ) and the anode placed $4 \mathrm{~mm}$ above the cathode and biased by a high voltage source measurement unit (SMU). The laser pulse hits the cathode at a $84^{\circ}$ glancing angle.

First, the laser energy is swept from $\sim 30 \mathrm{~nJ}$ to $10 \mu \mathrm{J}$ using $20 \mathrm{~dB}$ and $10 \mathrm{~dB}$ neutral density filters, while the anode voltage is kept constant. The signal collected is DC current 
through the cathode and anode resulting in average current measurements over a series of electron pulses with $3 \mathrm{kHz}$ repetition rate. The current is then converted to emitted charge per pulse by dividing the average current value by the repetition rate of the laser. Data is collected from varied anode voltages and incident energies.

\section{RESUlts}

The results from the sweeps are plotted in Fig. 3. At low intensities $(<0.1 \mu \mathrm{J})$, the electric field is not strong enough to significantly alter the potential barrier, requiring 3-4 photon absorption for electron emission from the high-aspect tips (power dependence slope of $\sim 3.9$ shown in Fig. 3). At laser intensities over $2 \mu \mathrm{J}$, the slope bends over to a $\sim 0.5$ power dependence, evidencing a transition to the tunneling emission regime (Fig. 3). This transition has been reported in [2] and [4].

At higher incident energies, the anode bias has a significant effect on the emitted charge. At $9.3 \mu \mathrm{J}$ incident energy, the emitted charge is $0.27 \mathrm{pC}$ with $10 \mathrm{~V}$ anode bias and $1.39 \mathrm{pC}$ with a $1000 \mathrm{~V}$ anode bias. The electric field from the anode bias is $3-4$ orders of magnitude lower than the field from the incident laser so there should be no enhancement from increase in anode bias. There are several possible explanations on the enhancement including charge blockage and an increase in the accumulation layer on the silicon surface. When the electrons are emitted in the short time scale, they produce a sheet of charge with the electric field pointing back towards the tips. This field is calculated to be on the same order as the anode bias thus possibility moving this sheet further away to allow for more electrons to be emitted [2]. The silicon emitters are ntype semiconductor with an accumulation layer on the surface interface with vacuum. The increase in anode bias before the laser pulse arrives will increase the available electrons at the surface to be emitted.

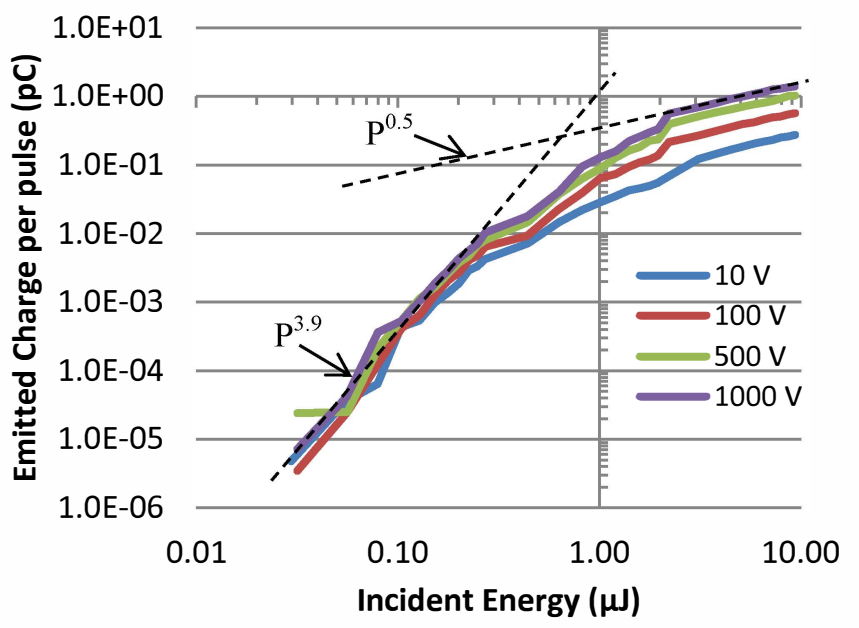

Fig. 3 - Incident laser energy sweeps at different anode biases. The results show multi-photon emission at low energies and then bending over to field emission

Fig. 4 shows three different cathode currents on three different sample locations with the beam being unblocked at time $0 \mathrm{~s}$ at an unexposed area. All three curves show stable current emission after 8 million pulses. The lowest curve in Fig. 4 has lower noise than the others and is slightly rising; this is because field emission current is highly non-linear with respect to the photon energy and the activation of the cathode takes longer at lower fields [2]. The highest curve in Fig. 4 shows an average $1.2 \mathrm{pC}$ electron emission (3.6 nA). SEM images taken after the 8 million pulses showed no visible damage. There was no measureable different between the nonexposed tips and the exposed tips from Fig 1a. When the energy of the laser pulses is over $20 \mu \mathrm{J}$, the electric field at the tips becomes too high, which oblates the tips, leaving a $\sim 85 \mu \mathrm{m}$ by $1800 \mu \mathrm{m}$ mark in the samples that matches the laser spot size (Fig. 1b).

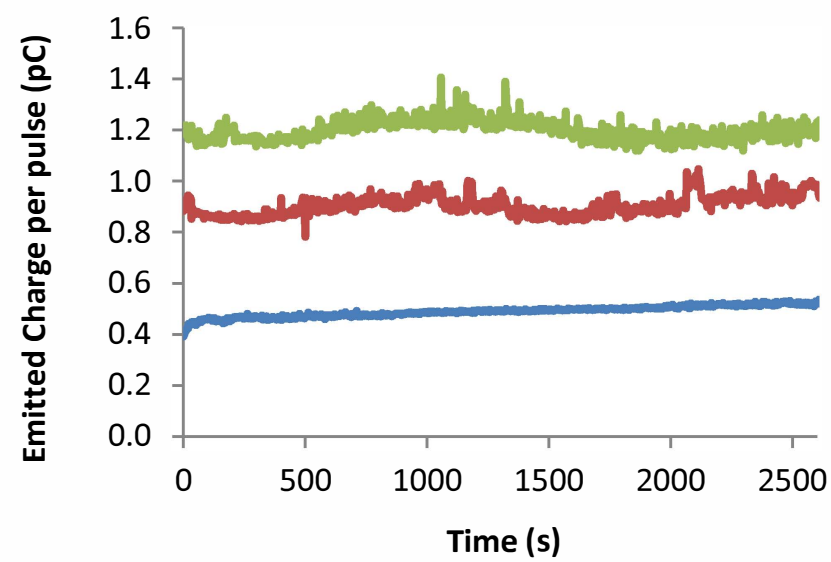

Fig. 4 - Stability of emitted current from the photocathode over time. Top green line is $9.5 \mu \mathrm{J}$ with $1000 \mathrm{~V}$ anode bias, middle red line is $9.5 \mu \mathrm{J}$ with $500 \mathrm{~V}$ anode bias and the bottom blue line is $3.0 \mu \mathrm{J}$ with $500 \mathrm{~V}$ anode bias.

\section{ACKNOLEDGEMENTS}

The device fabrication was done in the Microsystems Technology Laboratories, MIT. We would like the thank W. Graves, R. G. Hobbs and Y. Yang from MIT for the helpful discussions. This work was funded by the Defence Advanced Research Projects Agency / Microsystem Technology Office (DARPA/MTO) under contract N66001-11-1-4192 (program managers J. Mangano and D. Palmer). Any opinions, findings, and conclusions or recommendations expressed in this publication are those of the authors and do not necessarily reflect the views of the US Government and therefore, no official endorsement of the US Government should be inferred.

\section{REFERENCES}

[1] W. S. Graves, F. X. Kärtner, D. E. Moncton, and P. Piot, "Intense superradiant $\mathrm{X}$ rays from a compact source using a nanocathode array and emittance exchange," Phys. Rev. Lett., vol. 108, no. 26, p. 263904 , Jun. 2012.

[2] M. E. Swanwick, P. D. Keathley, F. X. Kärtner, and L. F. VelásquezGarcía, "Ultrafast Photo-Triggered Field Emission Cathodes using Massive Uniform Arrays of Nano-Sharp High-Aspect-Ratio Silicon Structures," to be presented to the $17^{\text {th }}$ International Conference on Solid-State Sensors, Actuators, and Microsystems (Transducers 2013), Barcelona, Spain, June 16 - 202013.

[3] P. D. Keathley, A. Sell, W. P. Putnam, S. Guerrera, L. F. VelásquezGarcía, and F. X. Kärtner, "Strong-field photoemission from silicon field emitter arrays," Annalen der Physik, vol. 525, no. 1-2, pp. 144-150, 2013.

[4] G. Herink, D. R. Solli, M. Gulde, and C. Ropers, "Field-driven photoemission from nanostructures quenches the quiver motion," Nature, vol. 483, no. 7388, pp. 190-193, Mar. 2012.

\section{CONTACT}

*M. E. Swanwick tel.+1-617-3060851; swanwick@mit.edu 